\title{
Design of a Pseudo-Holographic Distributed Time-of-Flight Sonar Range-Imaging System.
}

\author{
Lee Streeter,* Jonathan Scott, Carl A. Lickfold, Michael J. Cree. \\ School of Engineering \\ The University of Waikato \\ Private Bag, 3105. \\ Hamilton, New Zealand \\ Email: streeter@waikato.ac.nz
}

\begin{abstract}
The design of an audible sonar distributed sensor time-of-flight range imaging system is investigated, sonar being chosen as a substitute for optical range imaging due to cost and simplicity of implementation. The distributed range imaging system proposed is based on the holographic principle where the sensors detect the self interference of the reflected sound from the scene, and the Fourier analysis computes the reflected object profile. An approximate linearised model used in related holographic imaging techniques is found to be inappropriate for the design, and qualitative assessment of simulations show that removing the linearisation dramatically improves image reconstruction. Quantitatively the nonlinear reconstruction improves the RMSE by a factor of 1.3-2.1 times. The full nonlinear reconstruction is slow, and mathematical development lead to 15 fold reduction in computation time.
\end{abstract}

Index Terms-Holographic imaging, sonar, time-of-flight, stepped frequency continuous wave.

\section{INTRODUCTION}

Time-of-flight (ToF) range imaging is the method of distance measurement by indirect measurement of the travel time of sound or light emitted from the source, backscattered by objects in the scene, and returned to the detector [1]. Optical ToF range imaging cameras perform optical ToF ranging at each pixel in an array of sensors by continuously modulating the light source and sensor, correlating the light return signal with the sensor modulation, and examining phase shift in the correlation. Strong limitations exist on ToF camera hardware, especially the rate at which the sensor can be modulated (up to $150 \mathrm{MHz}$ ). Recent results have highlighted advantages in increasing the bandwidth [2], viz. opening doors to frequency stepping and Doppler analysis for range and motion measurement. Therefore we seek alternative configurations for high bandwidth ranging. Optical hardware is technically challenging and expensive, whereas audio range sonar is relatively cheap and simple to implement. Furthermore the wavelength of audible sound waves is comparable to the amplitude modulated wavelength of light. Therefore, we investigate a sonar ranging configuration as a model for the development and testing of algorithms for a potential optical system.

ToF range imaging has grown into an important topic of study, with strong commercial interest and broad applications in shape measurement, motion analysis, and medical imag-

978-1-5090-2748-4/16/\$31.00 (C)2016 IEEE ing [1]. Prominent examples of amplitude modulated continuous wave $(\mathrm{AMCW}) \mathrm{ToF}$ cameras are the Microsoft Kinect V2 [3], the range of cameras from PMD technologies ${ }^{1}$ and the Softkinetic depthsense cameras ${ }^{2}$. Most commonly, ToF range imaging is performed in the optical domain (including infrared) of photonic energy using specialised imaging hardware. Other technologies are included, such as microwave energy [4] and sonar [5]-[7]. These other techniques have advantages over optical imaging in that the energy can travel through media that photonic energy in the optical band cannot. Sonar, in particular the audio range, requires cheap components and the generation of waveforms at much lower frequencies than for optical modulation, hence is straightforward to use and experiment with.

Phased array systems based on distributed sources or detectors typically focus on beamforming such that either the transmitted beam, or the virtual beam seen by the receivers, is steerable [7], [8]. Imaging then is performed by steering the beam to a number of directions of interest, and measuring the response for each. The process is practical, but requires a time consuming scanning procedure. Coding, e.g Walsh coding, via amplitude modulation also assists in virtual beamforming, which has been demonstrated in a distributed ultrasonic range imaging system [9], [10]. In contrast, holographic imaging runs the phased array principle in reverse. A point source of energy is transmitted to the scene, which is backscattered to an array of detectors that sample the resultant interference pattern. The inverse problem then is to determine the scene that caused the measurements. Examples include coherent optical holographic lensless imaging [11], [12] using tunable lasers, and tomographic mammography using microwave radio [4]. Holographic ultrasound sonar for underwater applications has a long history [5], [6] where traditional sonar techniques like the so-called A-mode and C-mode (respectively depth and scanned sonar), with continuous frequency modulation and acoustic lenses, were used.

In this paper we apply the holographic imaging principle to sonar imaging, and combine the holographic technique

\footnotetext{
${ }^{1}$ http://www.pmdtec.com/, date retrieved 12/9/2016.

${ }^{2}$ https://www.softkinetic.com/Products/DepthsenseCameras, date retrieved 12/9/2016.
} 
with stepped frequency continuous wave (SFCW) ranging. The proposed system is a coherent sonar model of a potential amplitude modulated (AM) optical system, where the equipment for audible sonar is much cheaper and easier to design than the equivalent AM optical equipment. The sonar system is suitable for undergraduate student teaching, both as a design and a research project. We develop the theory in Section II and consider the design parameters for accurate reconstruction of a simple spatial pattern in Section III. In Section III we also discuss practical hardware configurations. Simulation demonstration is described in Section IV and results are given and discussed in Section V.

\section{THEORY}

The ToF principle of ranging is based on the detection of phase shifts in some waveform. The size of the phase shift is dependent on the the combination of wave frequency and flight time, the ToF, of the wave. In the case of sound, the wave travels at a nominal speed of $c=343 \mathrm{~m} / \mathrm{s}$ in air. The generation of sound waves with a loud speaker of frequencies in the range $1-20 \mathrm{kHz}$ is straightforward with standard benchtop function generators and amplifiers. Detection of such waves is equally simple with easily obtained microphone and amplifier components.

Consider a sound wave of frequency $f$. If the wave travels distance $d$ from speaker to microphone then the received waveform observed over time, $t$, is modelled as

$$
r(t)=\alpha \sin \left(2 \pi f t-\frac{2 \pi f d}{c}\right) .
$$

The signal from the microphone is amplified and then passed through a mixer, a switching component, where the switch is opened and closed at frequency $f$. This process is called homodyne demodulation [1], which is modelled by the multiplication

$$
\begin{aligned}
m_{0}(t ; f, d) & =r(t) \sin (2 \pi f t) \\
= & \frac{\alpha}{2} \cos \left(\frac{2 \pi f d}{c}\right)-\frac{\alpha}{2} \cos \left(4 \pi f t-\frac{2 \pi f d}{c}\right) .
\end{aligned}
$$

Passing the demodulated signal through a filter, or equivalently integrating for a period $T \gg 1 / f$, removes the second cosine term in Eqn. 2 leading to the single point measurement model

$$
m(f, d)=\frac{\alpha T}{2} \cos \left(\frac{2 \pi f d}{c}\right) .
$$

Eqn. 3 is called the correlation waveform [1].

To determine the distance, $d$, we need to vary some parameter in the correlation waveform to build up data conducive to analysis to compute $d$. To such end we step $f$ to each of a set of discrete values whilst continuously operating the speaker-microphone-mixer system. Measurements are taken of $m(f, d)$ for each fixed value of $f$ : the so-called stepped frequency continuous wave (SFCW) method [2]. SFCW then returns $m(f, d)$ as a cosine waveform of frequency $2 \pi d / c$, and finding the peaks in the Discrete Fourier transform (DFT) readily gives $d[2],[13]$.
A standard microphone receives sound over a wide solid angle, and over distances that range as far as the sound can travel out and back without excessive attenuation (up to ten metres of detection range is easily achieved). Therefore the measured waveform consists of the sum of all returns within range of the sonar system, and the measurement represented in Eqn. 3 must be modified to reflect the integration of sound over all possible travel paths. To such end let $\mathbf{p}=\left(p_{x}, p_{y}, p_{z}\right)$ be the three dimensional vector of positions with respect to the origin that the sound can travel to, $d=2|\mathbf{p}|, \alpha=2 A(\mathbf{p}) / T$ be the reflectance profile over $\mathbf{p}$, and assuming that the microphone is at the same position as the speaker, the measurement process becomes the three dimensional volume integral

$$
m(f)=\int_{V} A(\mathbf{p}) \cos \left(\frac{4 \pi f|\mathbf{p}|}{c}\right) d \mathbf{p},
$$

In the special case that we have a single reflecting point object then $A(\mathbf{p})$ is a Dirac delta function and we recover Eqn. 3, up to multiplicative constants.

Position the speaker at the origin, and move the microphone to a separate position from the origin represented by the vector r. The sound must now travel the distance $|\mathbf{p}|$ to the scene and then distance $|\mathbf{p}-\mathbf{r}|$ back to the microphone, and Eqn. 4 becomes

$$
m(f, \mathbf{r})=\int_{V} A(\mathbf{p}) \cos \left(\frac{2 \pi f|\mathbf{p}|}{c}+\frac{2 \pi f|\mathbf{p}-\mathbf{r}|}{c}\right) d \mathbf{p} .
$$

Eqn. 5 describes the self interference of the returning sound wave at each microphone detector point $\mathbf{r}$. It is this self interference that encodes the surface reflectance pattern in $m(f, \mathbf{r})$ such that it can be recovered by appropriate processing. Therefore we call Eqn. 5 the sonar pseudo-holographic imaging model. Assuming that $|\mathbf{p}| \gg|\mathbf{r}|$, then

$$
|\mathbf{p}-\mathbf{r}| \approx|\mathbf{p}|-\hat{\mathbf{p}} \cdot \mathbf{r},
$$

where $\hat{\mathbf{p}}$ is the unit vector of $\mathbf{p}$, and

$$
m(f, \mathbf{r}) \approx \int_{V} A(\mathbf{p}) \cos \left(\frac{4 \pi f|\mathbf{p}|}{c}-\frac{2 \pi f \hat{\mathbf{p}} \cdot \mathbf{r}}{c}\right) d \mathbf{p} .
$$

Expansion of the cosine gives

$$
\begin{aligned}
m(f, \mathbf{r}) & \approx \int_{V} A(\mathbf{p}) \cos \left(\frac{4 \pi f|\mathbf{p}|}{c}\right) \cos \left(\frac{2 \pi f \hat{\mathbf{p}} \cdot \mathbf{r}}{c}\right) d \mathbf{p} \\
& +\int_{V} A(\mathbf{p}) \sin \left(\frac{4 \pi f|\mathbf{p}|}{c}\right) \sin \left(\frac{2 \pi f \hat{\mathbf{p}} \cdot \mathbf{r}}{c}\right) d \mathbf{p} .
\end{aligned}
$$

Inversion of Eqn. 8 to recover $A(\mathbf{p})$ is performed by Fourier analysis. The exact details of the inversion depend on the design of the sensing system, which is the topic of the next section.

\section{DESIGN}

The electronic design required to implement a sonar system for indoor short range distance measurement is straightforward and uses readily available components. Proof of concept design and development is underway. A Tektronix (Beaverton, Oregon, USA) 3021 arbitrary function generator (AFG) is 
computer controlled over a serial port using SCPI commands and data is read into the computer using a LabJack (Lakewood, Colorado, USA) U3 analogue to digital (ADC) converter. The control of the AFG and A2D is handled using Matlab (The Mathworks, Natick, Massachusetts, USA). The signal from the AFG is split and amplified, one path going to a tweeter loudspeaker capable of $20 \mathrm{kHz}$ and the other to a Texas Instruments TS5A23157QDGSRQ1 Analogue Switch that acts as the receive mixer. A PUI Audio (Dayton, Ohio, USA) AOM-6378L-R Microphone receives the audio signal, which is amplified and then mixed by the switching circuit. The output of the mixer is low-pass filtered and digitised by the A2D.

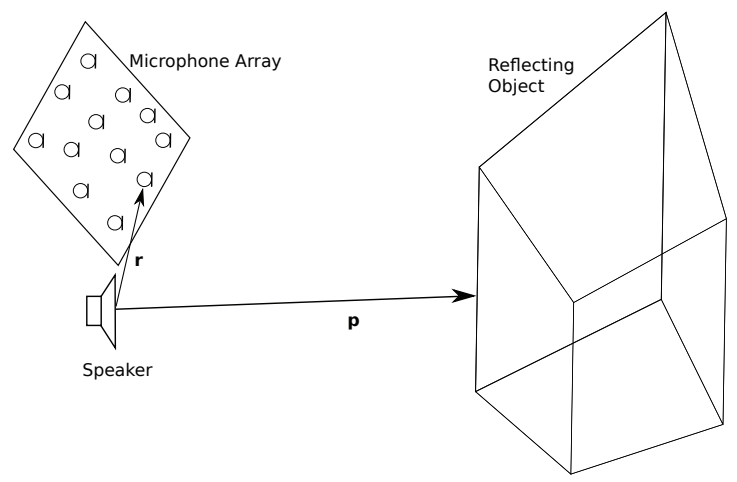

Fig. 1. Diagram of the sonar pseudo-holographic imaging system. Sound emanates from the speaker at a given frequency, and the microphone array sample the reflected interference pattern from objects in the scene. The origin is assumed to be at the speaker and the vectors $\mathbf{p}$ and $\mathbf{r}$ are indicated as arrows.

The more challenging design questions are to determine the operational parameters required to obtain data from which the return profile can be reconstructed. Namely the microphone arrangement, the sound frequency bandwidth and step size, and the working distance must all be carefully chosen. The frequency step size determines the maximum working distance beyond which the correlation waveform aliases and objects will appear closer that what they actually are. The frequency bandwidth determines the minimum resolvable distance between distances from the speaker, but for a fixed step size increasing the bandwidth necessitates more steps. Ideally data acquisition should be fast, so there is a tradeoff between data quality and data acquisition time. Similarly spreading the microphones over a wider area leads to better discrimination between reflectors but also increases the possibility that the approximation in Eqn..6 becomes inaccurate.

In practice an array of microphones is arranged over a plane [4], so the vector $\mathbf{r}=\left(r_{x}, r_{y}, 0\right)$, which spans a twodimensional space. The two-dimensional Fourier transform over the surface $S$ spanned by $\mathbf{r}$ recovers the complex phasor return pattern up to the Fourier projection slice theorem [13]

$$
\begin{aligned}
& \int_{S} m(f, \mathbf{r}) e^{-i 2 \pi \mathbf{k} \cdot \mathbf{r}} d \mathbf{r} \\
\approx & \int_{V} \int_{S} A(\mathbf{p}) \cos \left(\frac{4 \pi f|\mathbf{p}|}{c}\right) \cos \left(\frac{2 \pi f \hat{\mathbf{p}} \cdot \mathbf{r}}{c}\right) e^{-i 2 \pi \mathbf{k} \cdot \mathbf{r}} d \mathbf{p} d \mathbf{r}
\end{aligned}
$$

$$
\begin{aligned}
& -\int_{V} \int_{S} A(\mathbf{p}) \sin \left(\frac{4 \pi f|\mathbf{p}|}{c}\right) \sin \left(\frac{2 \pi f \hat{\mathbf{p}} \cdot \mathbf{r}}{c}\right) e^{-i 2 \pi \mathbf{k} \cdot \mathbf{r}} d \mathbf{p} d \mathbf{r} \\
& =\frac{1}{2} \int_{V} A(\mathbf{p}) \cos \left(\frac{4 \pi f|\mathbf{p}|}{c}\right)\left(\delta\left(\hat{\mathbf{p}}_{2}+\mathbf{k}\right)+\delta\left(\hat{\mathbf{p}}_{2}-\mathbf{k}\right)\right) d \mathbf{p} \\
& -\frac{i}{2} \int_{V} A(\mathbf{p}) \sin \left(\frac{4 \pi f|\mathbf{p}|}{c}\right)\left(\delta\left(\hat{\mathbf{p}}_{2}+\mathbf{k}\right)-\delta\left(\hat{\mathbf{p}}_{2}-\mathbf{k}\right)\right) d \mathbf{p} \\
& =\int_{-\infty}^{\infty} A(\hat{\mathbf{p}})\left\{\cos \left(\frac{4 \pi f|\mathbf{p}|}{c}\right) \pm i \sin \left(\frac{4 \pi f|\mathbf{p}|}{c}\right)\right\} d p_{z}
\end{aligned}
$$

where $\hat{\mathbf{p}}_{2}=\left(p_{x}, p_{y}\right) /|\mathbf{p}|$, and the Dirac delta functions that arise from the FT of sine and cosine allow us to equate $\mathbf{k}$ in the last line with $\mathbf{p}$. The \pm occurs from taking the FT of a real signal, manifesting as both positive and negative frequencies. Note the loss of distance specificity in Eqn. 9. This loss of distance information is the reason for using SFCW, namely that by the final step of Fourier analysis over $f$ we recover the distance profile.

The minimum resolvable distance $\Delta d$ in SFCW is related to the bandwidth $B$ by [2]

$$
\Delta d=\frac{c}{2 B}
$$

Similarly if the step between frequencies is $\Delta f$ the ambiguity distance, the maximum unambiguous measurement distance, is

$$
d_{\max }=\frac{c}{2 \Delta f}
$$

The factor of $\frac{1}{2}$ arises from the use of real signals giving rise to a negative frequency component which which will alias for distances greater than $d_{\max }$. However, if quadrature is obtained [14] then we can form the corresponding complex number, the negative frequency vanishes, and the ambiguity distance doubles. The spacing of microphones also has implications for the resolving power of the imaging system. Namely, if the microphones are spread out over distance $x$ in a given dimension orthogonal to $z$ then then the minimum resolvable transverse distance, $\Delta x$, in that dimension is

$$
\Delta x=\frac{c}{2 B x} .
$$

A less obvious parameter choice to make is what frequencies to use in SFCW, in other words at what frequency $f_{0}$ should the modulation begin at (where the final frequency is $\left.f_{0}+B\right)$. From the above arguments there is prima facie no distinction between choices of $f_{0}$. However, a slightly deeper examination of Eqn. 5 reveals that a high frequency allows us to observe more periods of the spatial interference pattern over the detectors than a low frequency will. More periods facilitates separation of returns, improving the resolving power of the system.

Returning to Eqn. 12, clearly widening the extent of the detector positions, $x$, improves the theoretical image quality, assuming that the linearity assumption of Eqn. 6 holds. However, increasing $x$ reduces the accuracy of the approximation in Eqn. 6, resulting in a tradeoff in design parameters. To 
circumvent this tradeoff we consider the following alternative to the FT [5], [6]

$$
\begin{array}{r}
\int_{f=f_{0}}^{f_{0}+B} \int_{S} m(\mathbf{r}, f) e^{-\frac{i 2 \pi f}{c}(|\mathbf{p}|+|\mathbf{p}-\mathbf{r}|)} d \mathbf{r} d f \\
=\int_{f=f_{0}}^{f_{0}+B} \int_{S} \int_{V} A(\mathbf{p}) \cos \left(\frac{2 \pi f}{c}(|\mathbf{p}|+|\mathbf{p}-\mathbf{r}|)\right) \\
\times e^{-\frac{i 2 \pi f}{c}\left(\left|\mathbf{p}^{\prime}\right|+\left|\mathbf{p}^{\prime}-\mathbf{r}\right|\right)} d \mathbf{p} d \mathbf{r} d f,
\end{array}
$$

in which we do not make any approximations. Using formal interchange to take the integral over $f$ first we have

$$
\begin{gathered}
\int_{S} \int_{V} A(\mathbf{p}) \int_{f=f_{0}}^{f_{0}+B} \cos \left(\frac{2 \pi f}{c}(|\mathbf{p}|+|\mathbf{p}-\mathbf{r}|)\right) \\
\times e^{-\frac{i 2 \pi f}{c}\left(\left|\mathbf{p}^{\prime}\right|+\left|\mathbf{p}^{\prime}-\mathbf{r}\right|\right)} d f d \mathbf{p} d \mathbf{r} \\
=\frac{1}{2} \int_{S} \int_{V} A(\mathbf{p}) \delta\left(|\mathbf{p}|+|\mathbf{p}-\mathbf{r}| \pm\left|\mathbf{p}^{\prime}\right| \pm\left|\mathbf{p}^{\prime}-\mathbf{r}\right|\right) d \mathbf{p} d \mathbf{r} \\
=A\left( \pm \mathbf{p}^{\prime}\right)
\end{gathered}
$$

In other words, we reconstruct the complex waveform to each point in space as seen by each detector, and rely on the principle of orthogonality between waves of different frequency to spatially separate the returns. Like before, the \pm term represents the presence of both the positive and negative frequency in the real cosine, and if quadrature is obtained then the negative frequency vanishes.

The reconstruction represented in the left hand side of Eqn. 13 requires $\mathcal{O}\left((m n o)^{2}\right)$ operations to digitally estimate the nested two spatial integrals and the one integral over frequency. Therefore a more efficient, hence faster, algorithm is required. Expansion of the exponential and factorisation yields

$$
\int_{f=f_{0}}^{f_{0}+B} e^{-\frac{i 2 \pi f}{c}|\mathbf{p}|} \int_{S} m(\mathbf{r}, f) e^{-\frac{i 2 \pi f}{c}|\mathbf{p}-\mathbf{r}|} d \mathbf{r} d f .
$$

The inner integral is a convolution, and Eqn. 15 may be written as

$$
\int_{f=f_{0}}^{f_{0}+B} e^{-\frac{i 2 \pi f}{c}|\mathbf{p}|}\left(\delta\left(p_{z}\right) m(\mathbf{p}, f) * e^{-\frac{i 2 \pi f}{c}|\mathbf{p}|}\right) d f
$$

where $*$ denotes convolution. The complex exponential term is precomputed for all spatial positions of interest. The convolution is performed using the Fourier convolution theorem, which in implementation is accelerated by leveraging the fast Fourier transform. The final integral over $f$ has the nonlinear operation of taking the magnitude $|\mathbf{p}|$, and does not obviously lend itself to use of the fast Fourier transform. Therefore, the more efficient method requires $\mathcal{O}\left(m^{2} n o \log (n) \log (o)\right)$ operations.

\section{Simulation}

In simulation we test the following.

- Linear approximate modulation: the modulation is modelled using Eqn. 6, viz. $I(\mathbf{p}) \cos \left(\frac{2 \pi f}{c}(2|\mathbf{p}|-\mathbf{p} \hat{\cdot} \mathbf{r})\right)$, and the image is reconstruction using the DFT.
- Linear image reconstruction: the data acquisition model follows Eqn. 5, assuming the linearisation approximation of Eqn. 6 is accurate, and the image reconstruction is performed using the DFT.

- Nonlinear reconstruction: the reconstruction is performed using Eqn. 13.

Two bandwidths are tested, $B=2 \mathrm{kHz}$ and $B=10 \mathrm{kHz}$. In both cases the highest frequency simulated is $f_{0}+B=20 \mathrm{kHz}$, a reasonable frequency limit to expect from moderately cheap audio equipment. The frequency step used is $\Delta f=2 \mathrm{~Hz}$, again easily achievable with standard benchtop AFG devices.

For visualisation purposes we perform the simulations in $2 \mathrm{D}$, with $\mathbf{r}$ reduced to one axis. The microphones are evenly spread out over an extent $x$, with the speaker placed at the centre. For the $2 \mathrm{kHz}$ bandwidth, $x$ is varied to the values $0.5,1$, and $2 \mathrm{~m}$. At the $10 \mathrm{kHz}$ bandwidth $x=2 \mathrm{~m}$ is tested. In the simulations 255 microphones are used, and the simulated demodulated signals are processed as raw. However, in practice more sophisticated techniques can be used [4]. For example when quadrature is acquired for each frequencydetector combination, the detectors can be placed randomly (or in a spiral for a 2D arrangement). The correlation between the detector signals gives the phase differences between each pair of microphones, which is then used in the image reconstruction. We also test the effect of aliasing due to the number of detectors in the nonlinear reconstruction. Over the $2 \mathrm{kHz}$ bandwidth we test $2^{N}-1$ detectors for $N \in\{4,5,6\}$.

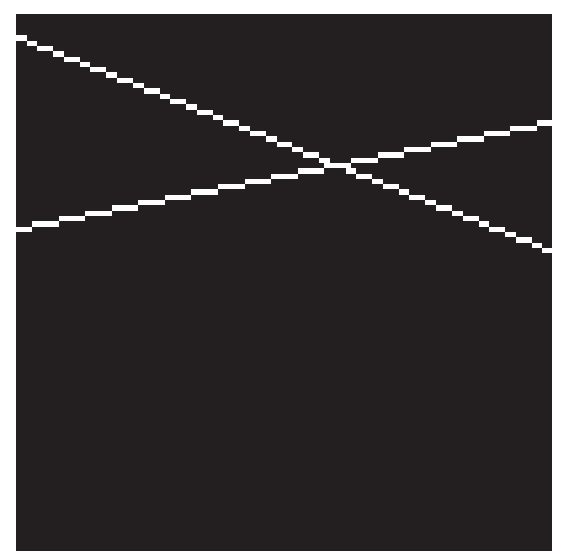

Fig. 2. Simulation 'surface' in 2D. The origin (and speaker) is at the bottom left. The spatial dimensions are $1 \mathrm{~m}$ horizontal and $5 \mathrm{~m}$ vertical.

The simulated scene is presented in Fig. 2. The spatial resolution is $1 \mathrm{~cm}$ per pixel horizontally and $5 \mathrm{~cm}$ per pixel vertically. The model test surface is motivated by two common test scenes in ToF imaging, the interior corner scene (e.g. a wall at right angles to the floor [1]), and the transparent foreground object and opaque background scene [15].

\section{RESUlts AND Discussion}

In Table I the RMS errors between the reconstructed and true simulated reflectance profile are given for the various test cases. The RMS errors are ordered from worst to best performance. In each case, the nonlinear reconstruction performed 
TABLE I

ERROR IN ARBITRARY UNITS (AU) OF THE RECONSTRUCTION FOR THE VARIOUS SIMULATION PARAMETERS. L-L REFERS TO LINEAR APPROXIMATE MODULATION, L-NL TO LINEAR IMAGE RECONSTRUCTION, AND NL-NL TO NONLINEAR RECONSTRUCTION.

\begin{tabular}{c|ccc} 
RMSE $(\mathrm{AU})$ & L-L & L-NL & NL-NL \\
\hline & & & \\
$B=2 \mathrm{kHz}, x=0.5$ & 0.32 & 0.32 & 0.15 \\
$B=2 \mathrm{kHz}, x=1$ & 0.23 & 0.25 & 0.12 \\
$B=2 \mathrm{kHz}, x=2$ & 0.17 & 0.19 & 0.12 \\
$B=10 \mathrm{kHz}, x=2$ & 0.15 & 0.14 & 0.11
\end{tabular}

the best. This agrees with qualitative inspection of the images of the reconstructed profiles, Figs. 3-6. The linear approximate modulation gives a best case scenario assuming that Eqn. 6 holds, but is unrealistic in any practical case. The linear image reconstruction case is more physically realistic, and these results, especially Figs. 5 and 6 indicate that using the Fourier transform to reconstruct the reflectance profile from distributed sensors is not appropriate. In particular, the use of the Fourier transform leads to the aforementioned tradeoff between detector extent and reconstruction quality. Namely, a smaller detector array extent maintains voracity of Eqn. 6, but a wider extent improves resolution, and qualitativly there does not appear to be a optimal operating point that gives a good image reconstruction. In contrast, the nonlinearised reconstruction, Eqn. 13, does not make any approximations, and as such does not suffer from the tradeoff that the Fourier transform does. Resultantly the reconstruction quality improves with increased detector extent.
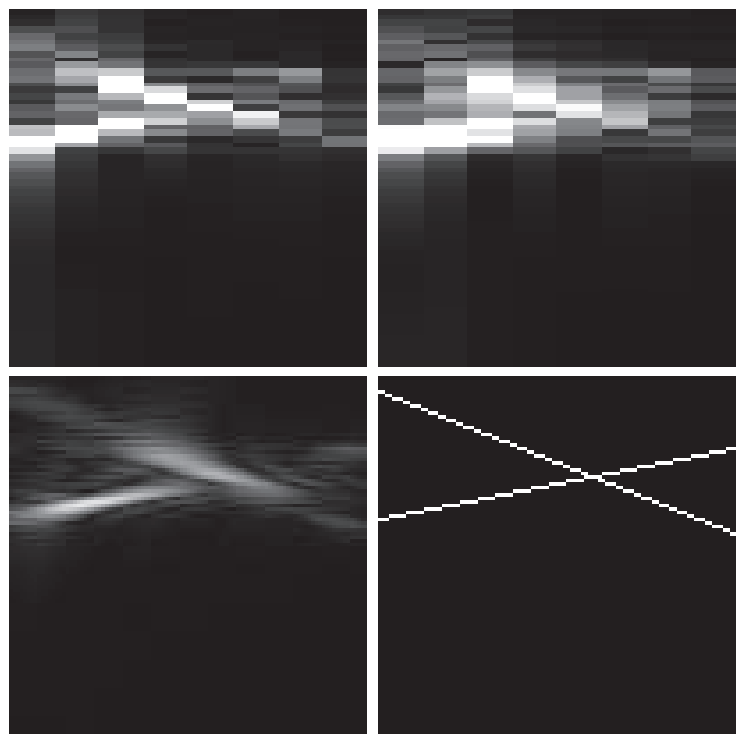

Fig. 3. Simulation results for $2 \mathrm{kHz}$ bandwidth and $0.5 \mathrm{~m}$ detector extent. The images are (top left) linear approximate modulation, (top right) linear image reconstruction, (bottom left) nonlinear reconstruction, (bottom right) true surface profile.

The effect of the number of detectors is shown in Fig. 7. Aliasing is apparent for 15 and 31 detectors over the $2 \mathrm{~m}$ extent, and is not present for 63 detectors. For the 15 detector experiment, the aliasing of the intersection of the two lines
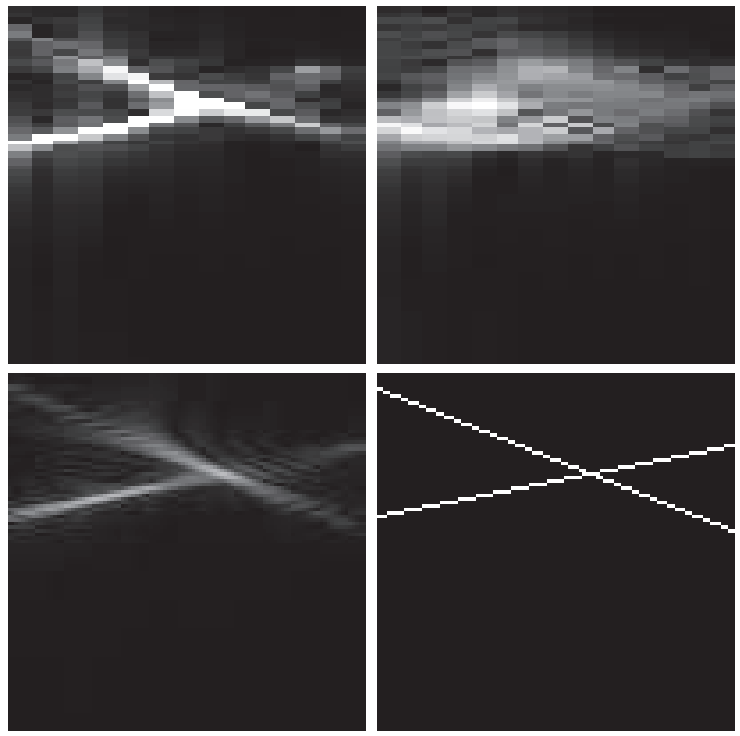

Fig. 4. Simulation results for $2 \mathrm{kHz}$ bandwidth and $1 \mathrm{~m}$ detector extent. The images are (top left) linear approximate modulation, (top right) linear image reconstruction, (bottom left) nonlinear reconstruction, (bottom right) true surface profile.
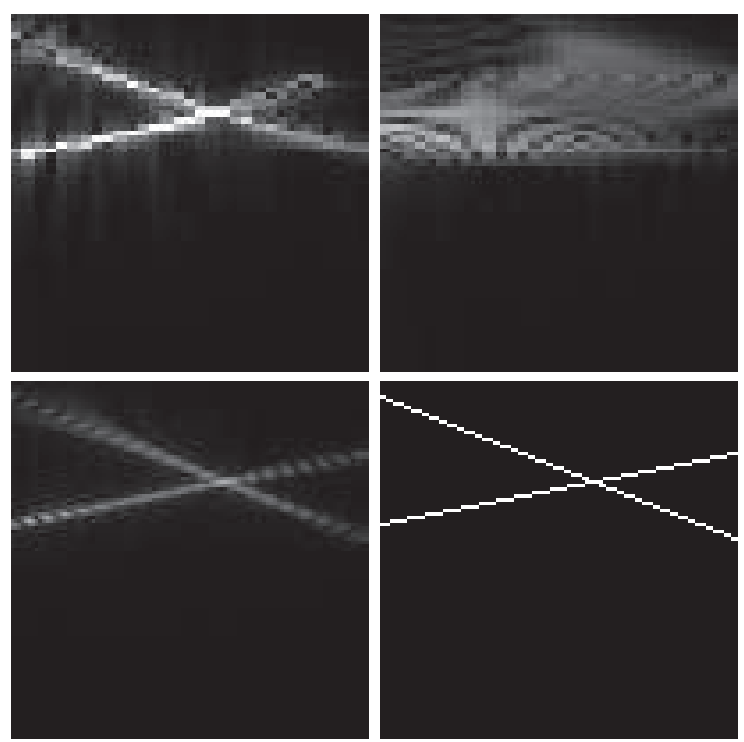

Fig. 5. Simulation results for $2 \mathrm{kHz}$ bandwidth and $2 \mathrm{~m}$ detector extent. The images are (top left) linear approximate modulation, (top right) linear image reconstruction, (bottom left) nonlinear reconstruction, (bottom right) true surface profile.

appears on the left of the image, indicating that the spacing between the aliases is less than the $1 \mathrm{~m}$ width of the scene. Under linear Fourier theory this spacing should be $\frac{2^{N}-1}{x}=$ $\frac{15}{2}=7.5 \mathrm{~m}$. Clearly the nonlinear reconstruction does not follow classical Fourier aliasing rules. A full mathematical analysis is left for future work.

Finally we note that, on a 2014 MacBook Pro equipped with a $2.8 \mathrm{GHz}$ Intel Core i7 cpu and $16 \mathrm{~GB}$ of ram, for the 1001 frequencies over the band $18-20 \mathrm{kHz}$ and 255 detectors the direct implementation of the nonlinearised reconstruction 

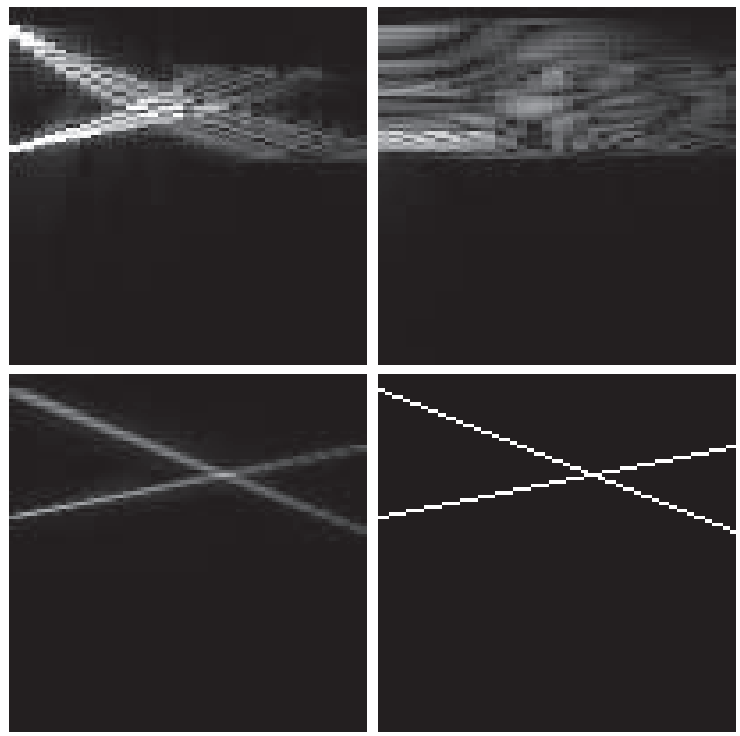

Fig. 6. Simulation results for $10 \mathrm{kHz}$ bandwidth and $2 \mathrm{~m}$ detector extent. The images are (top left) linear approximate modulation, (top right) linear image reconstruction, (bottom left) nonlinear reconstruction, (bottom right) true surface profile.
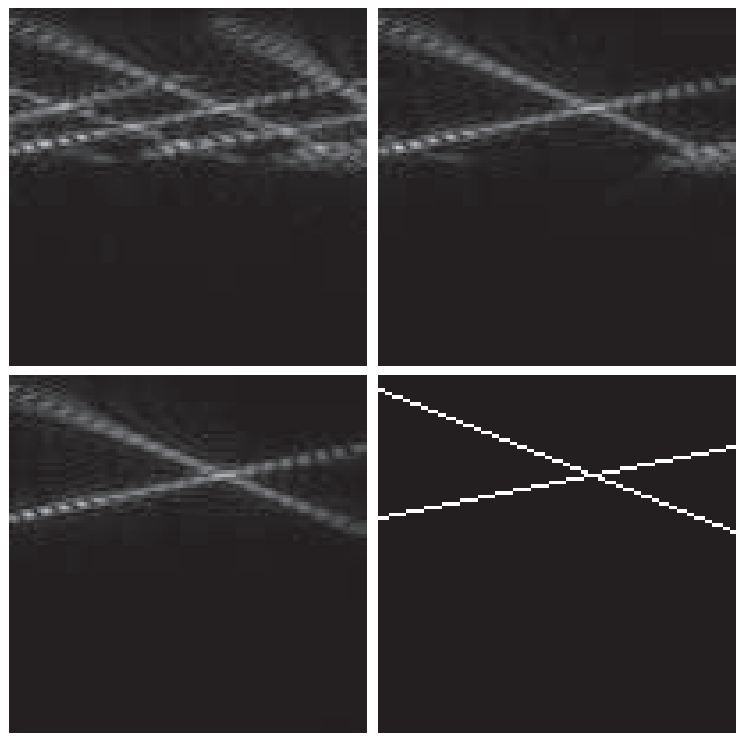

Fig. 7. Effect of aliasing. The images are (top left) $N=4$ (15 detectors), (top right) $N=5$ (31 detectors), (bottom left) $N=6$ (63 detectors), and (bottom right) the true surface profile.

required 185 seconds. The faster algorithm, by direct implementation of Eqn. 16, using the FFT to perform the required convolutions required 12 seconds, approximately 15 times faster.

\section{CONCLUSION}

We have explored the design of a distributed sonar ranging system using stepped frequency radar principles. The proposed sonar system is a model of a potential optical system, facilitating investigations into design configurations and processing algorithms with simple electronic design and cheap electronic components. The resultant design is suitable for an undergraduate project.

A far-field approximation that is used in microwave tomography that leads to fast image reconstruction by Fourier transform was investigated. Simulations revealed that the approximation leads to a tradeoff in design parameters that had no clear optimal operating point. Therefore the farfield approximation was found to be inappropriate. Removing that approximation leads to a nonlinearised reconstruction algorithm that relieved the system of tradeoffs, resulting in much superior image reconstruction, albeit at the cost of longer processing times. Mathematical development of the reconstruction algorithm improved efficiency. In simulation the processing time was successfully reduced by a factor of 15.

\section{REFERENCES}

[1] M. Grzegorzek, C. Theobalt, R. Koch, and A. Kolb, Eds., Tome-of-Flight and Depth Imaging. Berlin, Germany: Springer, 2013.

[2] R. Whyte, L. Streeter, M. J. Cree, and A. A. Dorrington, "Application of lidar techniques to time-of-flight range imaging," Applied Optics, vol. 54, no. 33, pp. 9654-9664, 2015.

[3] C. S. Bamji, P. OConnor, T. Elkhatib, S. Mehta, B. Thompson, L. A. Prather, D. Snow, O. C. Akkaya, A. Daniel, A. D. Payne, T. Perry, M. Fenton, and V.-H. Chan, "A $0.13 \mathrm{~m}$ cmos system-on-chip for a $512 \times 424$ time-of-flight image sensor with multi-frequency photodemodulation up to $130 \mathrm{mhz}$ and $2 \mathrm{gs} / \mathrm{s}$ adc," IEEE JOURNAL OF SOLID-STATE CIRCUITS, vol. 50, no. 1, pp. 303-319, 2014.

[4] L. Wang, R. Simpkin, and A. M. Al-Jumaily, "3D breast cancer imaging using holographic microwave interferometry," in 27th Image and Vision Computing New Zealand, Dunedin, New Zealand, 2012, pp. 180-185.

[5] T. Sato, "Holographic sonar 3D underwater ultrasonic imaging," Japanese Journal of Applied Physics, vol. 20, pp. 229-231, 1981.

[6] - "Holographic sonar imaging and its real-time image reconstruction," Japanese Journal of Applied Physics, vol. 22, pp. 87-89, 1983.

[7] Y. Han, J. Fu, F. Zhou, X. Tian, and Y. Chen, "Distributed and parallel subarray beamforming for near-field 3d sonar imaging," in 2013 3rd International Conference on Computer Science and Network Technology (ICCSNT), Dalian, China, 2013, pp. 1021-1025.

[8] M. Skolnik, Ed., Radar Handbook. New York, USA: McGraw Hill, 2008, ch. Phased Array Radar Antennas.

[9] Y. Tamura and T. Akatsuka, Holographic Sonar Using Orthogonal Transmitting Pulses. Boston, MA: Springer US, 1989, pp. 753-760.

[10] Y. Tamura, Y. Aochi, S. Terasaki, O. Takano, T. Akatsuka, C. Ishihara, and N. Ishii, A Multiple Shots 3-D Holographic Sonar Using a Set of Orthogonalized Modulating Signals. Boston, MA: Springer US, 1993, pp. 737-743.

[11] J. C. Marron and K. S. Schroeder, "Three-dimensioanl lensless iamging using laser frequency diversity," Applied Optics, vol. 31, no. 2, pp. 255262, 1992.

[12] A. B. Mateo and Z. W. Barber, "Multi-dimensional, non-contact metrology using trilateration and high resolution fmcw ladar," Applied Optics, vol. 54, no. 19, pp. 5911-5916, 2015.

[13] R. J. M. II, Handbook of Fourier Analysis and Its Applications. Oxford, UK: Oxford University Press, 2009.

[14] M. Jankiraman, Design of Multi-Frequency CW Radars. Rayleigh, North Carolina, USA: SciTech Publishing Inc., 2007.

[15] A. Kadambi, R. Whyte, A. Bhandari, L. Streeter, C. Barsi, A. Dorrington, and R. Raskar, "Coded time of flight cameras: sparse deconvolution to address multipath interference and recover time profiles," $A C M$ Transactions on graphics (SIGGRAPH), vol. 32, no. 6, p. 167, 2013.

\section{ACKNOWLEDGMENT}

L. Streeter was supported by a Marsden Council Fast Start grant. 